Editor's Note: Toolboxes are a new, occasional feature in the Journal designed to briefly highlight a new method or a resource of general use in neuroscience or to critically analyze existing approaches or methods. For more information, see http://www. jneurosci.org/itoa.shtml.

\title{
Uniform Serial Sectioning for Transmission Electron Microscopy
}

\author{
Kristen M. Harris, ${ }^{1,2,3}$ Elizabeth Perry, ${ }^{2}$ Jennifer Bourne, ${ }^{1,2}$ Marcia Feinberg, ${ }^{3}$ Linnaea Ostroff, ${ }^{3}$ and Jamie Hurlburt ${ }^{2,3}$ \\ ${ }^{1}$ Section of Neurobiology and Center for Learning and Memory, University of Texas at Austin, Austin, Texas 78712, ${ }^{2}$ Department of Neurology, Synapses, \\ and Cognitive Neuroscience Center, Medical College of Georgia, Augusta, Georgia 30912, ${ }^{3}$ Program in Neuroscience and Biology Department, Boston \\ University, Boston, Massachusetts 02118
}

The transmission electron microscope (TEM) was first used approximately half a century ago to answer important neurobiological questions, showing unequivocally that neurons communicate via synaptic junctions (Palay and Palade, 1955; Gray, 1959). TEM usually requires that biological specimens be $<100 \mathrm{~nm}$ thick. These thin sections allow electrons to pass and stop only where stains delineate objects of interest. From the beginning, serial thin sections were visualized and reconstructed to provide a threedimensional context (Birch-Anderson,

Received Sept. 13, 2006; revised 0ct. 23, 2006; accepted 0ct. 23, 2006.

This work was supported by National Institutes of Health Grants NS21184, NS33574, and EB002170 (K.M.H.).Serial sectioning was first learned from a fellow student, Barbara McGuire, when K.M.H. was a student in the Neurobiology Course at the Marine Biological Laboratory, Woods Hole (1980; Woods Hole, MA) and evolved during a postdoctoral fellowship with Dennis Landis and John Stevens. The methods were refined with the help of research assistants and students while K.M.H. was on the faculty of Harvard Medical School at Children's Hospital (Boston, MA), Boston University (Boston, MA), and the Medical College of Georgia (Augusta, $G A)$. Input from numerous collaborators and colleagues has been helpful to delineate reliable methods; in particular we thank Josef Spacek, Joanne Buchanan, and Thomas Reese. We also thank Robert Smith for help preparing the figures and supplemental movies.

Correspondence should be addressed to Kristen M. Harris, Section of Neurobiology and Center for Learning and Memory, The University of Texas at Austin, 1 University Station Stop C7000, Austin, TX 78712-0805. E-mail: kharris@mail.clm.utexas.edu.

E. Perry's present address: Department of Cellular Biology and Anatomy, Electron Microscopy Core, Medical College of Georgia, Augusta, GA 30912-2630.

M. Feinberg's present address: Department of Health Sciences, Boston University, Boston, MA 02118.

L. Ostroff's present address: Center for Neural Sciences, New York University, New York, NY 10003.

DOI:10.1523/JNEUROSCI.3994-06.2006

Copyright $\odot 2006$ Society for Neuroscience $\quad 0270-6474 / 06 / 2612101-03 \$ 15.00 / 0$
1955; Bang and Bang, 1957; Spacek and Lieberman, 1974). These efforts were considered heroic because of the difficulty in collecting and photographing the serial sections and producing realistic threedimensional reconstructions. In neurobiology, there is a renewed interest in serial section TEM (ssTEM) (Stevens et al., 1980) to understand how synaptic structure is modified during changes in brain state (Ostroff et al., 2002; Knott et al., 2006). The resolution of ssTEM is needed to distinguish and measure accurately dendrites, axons, glial processes, synapses, and subcellular organelles such as synaptic vesicles, polyribosomes, and endosomal compartments. ssTEM is a labor intensive enterprise; hence, choosing when to use it is important. Confocal and multiphoton microscopy provide lower resolution images from living cells to assess whether labeled structures have changed location or size; however, the resolution is too low to identify, quantify, and measure the dimensions and composition of structures in brain neuropil. ssTEM is required for accurate identification and measurement of objects smaller than $250 \mathrm{~nm}$. A sample volume of 500-1000 $\mu \mathrm{m}^{3}$ spanning 250-500 serial thin sections is practical with sSTEM. Photomontaging can be used to enlarge these volumes but at a marked increase cost in time. Here, we describe methods optimized to produce uniform ribbons of serial thin sections.

We aim for a section thickness of 45-50 nm to minimize overlap among small structures, such as synaptic vesicles $(\sim 35 \mathrm{~nm})$ or narrow axonal or astroglial processes $(\sim 50 \mathrm{~nm})$ that would be obscured by neighboring structures in thicker sections. We routinely collect 200-300 serial electron microscopic (EM) sections; however, much longer series can be collected with these methods. A review of fixation and processing methods is beyond the scope of this paper; as an example, we describe the procedure for hippocampal slices fixed rapidly in mixed aldehydes in the presence of microwave irradiation (Jensen and Harris, 1989). Within $1 \mathrm{~d}$, the slices are embedded in 7\% agarose, dissected to a region of interest, and sliced with a vibrating blade microtome (Leica, Nussloch, Germany) at 70 $\mu \mathrm{m}$ (Fig. $1 a-c$ ) to permit osmium, uranyl acetate, or other en bloc stains to penetrate uniformly, while allowing visualization of regions of interest in the Epon block later (Fig. 1d). Epon is shaved off in 1-3 $\mu \mathrm{m}$ increments to a region near the stimulating electrode indentation on a neighboring section (Fig. $1 h$, dotted line). A test thick section is used to guide the trimming of the series trapezoid with a diamond trim tool to a height of $\sim 30 \mu \mathrm{m}$, a width of $\sim 100-200 \mu \mathrm{m}$, and a depth of 20-30 $\mu \mathrm{m}$ for stability, and with one side slanted for orientation (Fig. $1 f, g$ ). Ribbons of serial thin sections are cut and then retrieved on pioloform-coated slot grids. Lowicryl and some other resins are notoriously difficult to ribbon (Fig. $1 h$ ) because the hydrophilic sections fall apart; 
however, a few quick sprays of salonquality hair spray and overnight drying produces uniform continuous ribbons on the same trapezoid (Fig. 1i).

The goal always is to have perfectly uniform section thickness along fold-free ribbons for accurate quantitative analyses (Fig. 2a). A $35^{\circ}$ diamond knife minimizes section compression (Figs. 2b,c). The grids are coated within $24 \mathrm{~h}$ before section pickup to avoid having the Pioloform sag under the weight of the sections during pickup, which can create folds after drying (Fig. 2b). Pioloform that is coated too thickly or with an uneven flow rate altered apparent section thickness (Fig. 2e). Small holes in the Pioloform (Fig. 2f) are avoided by drying the coated slide in a small jar with desiccant. Saturated aqueous uranyl acetate and freshly prepared Reynolds lead citrate are both filtered through a $0.22 \mu \mathrm{m}$ syringe filter, and grids were stained in a $\mathrm{CO}_{2}$ - free environment to avoid dark precipitate over the surface of the sections (Fig. 2g). Microwaveenhanced infiltration of epoxy resins had been used to speed the process, however, infiltration was not always uniform, which resulted in cracks (Fig. $2 h$ ); hence, tissue blocks are now infiltrated in epoxy resins overnight and then cured for $48 \mathrm{~h}$ in a $60^{\circ} \mathrm{C}$ oven. The ribbon curved if the north and south edges of the trapezoid were not parallel (Fig. 2i). Uneven section thickness (Fig. 2j) is easily corrected by enclosing the ultramicrotome (Fig. $2 k$ ) to prevent air drafts and local temperature changes. Stopping and restarting the ribbon also produces a change in section thickness (e.g., third section from the edge of the knife in Fig. 1j). A video camera is used to monitor progress, and the enclosure doors are not opened until the desired ribbon length is achieved. These methods produce long, fold-free, and clean ribbons of serial thin sections with uniform section thickness. Dry grids are then loaded into grid cassettes and stored in gelatin capsules for repeated viewing and photography in a TEM. For detailed methods, refer to supplemental methods, movies, and figures (available at http:// www.jneurosci.org as supplemental material). In addition, consult http://SynapseWeb.org, http://synapses.bu.edu, or http:// clm.synapses.utexas.edu for updates on these methods and detailed methods for photography, alignment, reconstruction, and measurement in three dimensions.

New approaches are sought to achieve the information currently available only with ssTEM more rapidly. Recently, serial block-face-scanning EM (Briggman and
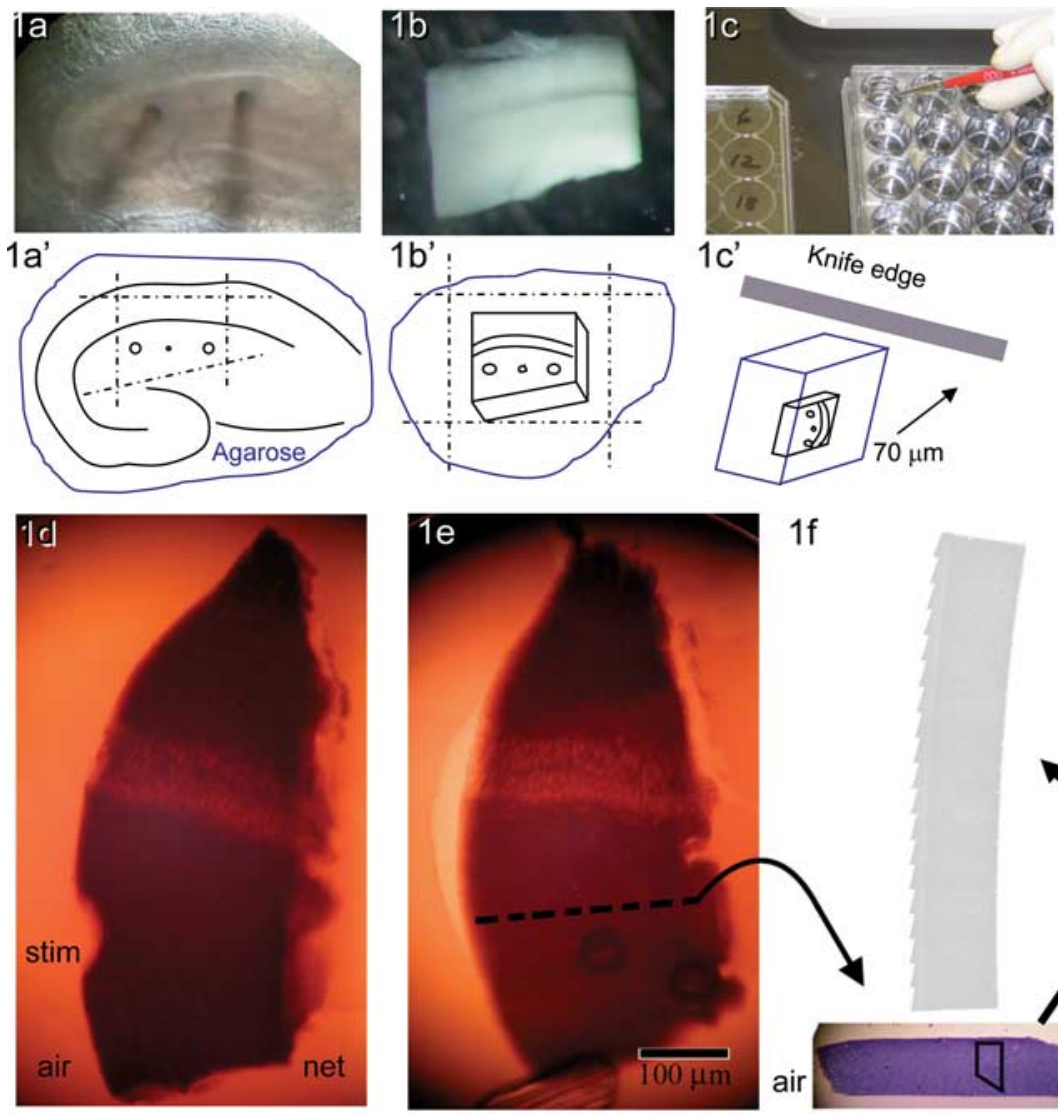

1f
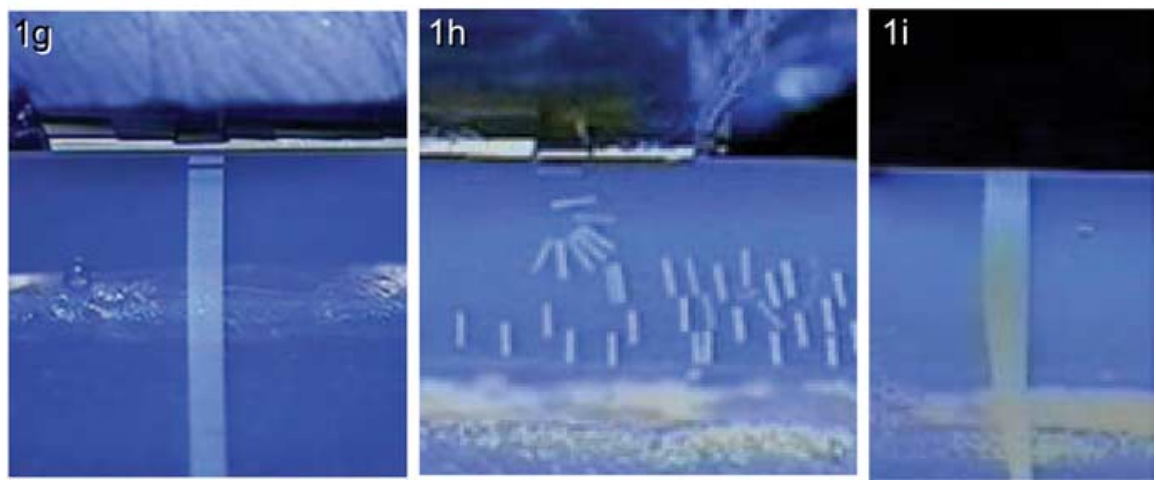

Figure 1. Preparation of serial thin sections from a specific region of interest. $\boldsymbol{a}$, Stimulating electrodes positioned in a hippocampal slice. $\boldsymbol{a}^{\prime}$, Diagram of a region of interest surrounding the indentations left by the stimulating electrodes on the slice, which is embedded in agarose for stability. $\boldsymbol{b}$, Fixed region of interest illustrated diagrammatically in $\boldsymbol{b}^{\prime}$ and turned on edge to obtain slices using a vibrating blade microtome to produce "vibra-slices" as illustrated in c. c, The 70 - $\mu \mathrm{m}$-section vibra-slices are gently transferred into a 24-well tissue culture plate containing $0.1 \mathrm{~m}$ phosphate buffer using a small brush at the corner of the surrounding agarose to avoid mechanically induced dark artifacts in the tissue. $d, 0$ n-edge view of a vibra-slice through the depth of the hippocampal slice from the air to the net surface; this vibra-slice is through the indentation left by the stimulating electrode (stim). $\boldsymbol{e}$, Neighboring vibra-slice used to obtain series near the stim, by shaving off the Epon to the dotted line. The scale bar in $\boldsymbol{e}$ is for $\boldsymbol{d}$ and $\boldsymbol{e}$. $\boldsymbol{f}$, Toluidine blue-stained thick section at the bottom is used to guide where to place the trapezoid on the face of the Epon block. The goal is to have a long ribbon of uniform section thickness. $\boldsymbol{g}$, Uniform ribbon sectioning, except where the section arm was stopped and restarted. $\boldsymbol{h}$, Routine Lowicryl embedded tissue does not ribbon well. $\boldsymbol{i}$, After hair spray, the same trapezoid sections well.

Denk, 2006) has been added to the EM toolkit. The advantage of this approach is that fragile, serial thin sections do not need to be handled; instead, the remaining block face is photographed after each section is removed. Theoretically, larger sample areas could be photographed without montaging; however, the resolution achieved at this lower magnification does not currently allow deciphering of individual axons, astroglial processes, dendritic spines, synapses, and their composition of organelles in brain neuropil. Automatic montaging, alignment, and 

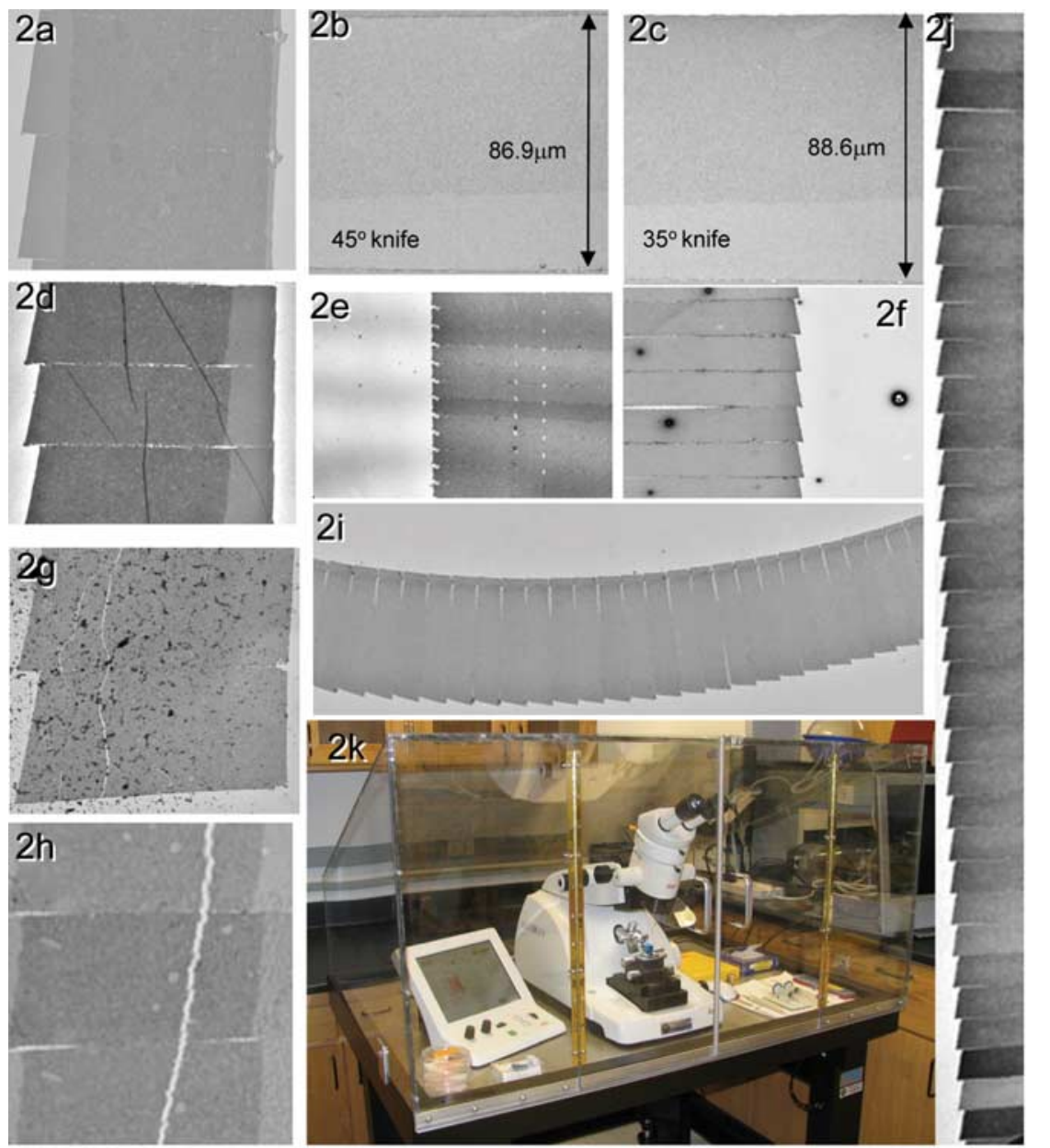

Figure 2. Problems to overcome during SSTEM. $\boldsymbol{a}$, Goal is a set of clean serial sections of uniform thickness. $\boldsymbol{b}, \boldsymbol{c}$, Section compression is minimized by using a $35^{\circ}$ diamond knife. $\boldsymbol{d}$, Folds in the Pioloform film. $\boldsymbol{e}$, Uneven Pioloform film thickness. $\boldsymbol{f}$, Holes in Pioloform film. $\boldsymbol{g}$, Dirty grid stain. $\boldsymbol{h}$, Poorly infiltrated tissue has cracks. $\boldsymbol{i}$, Curved ribbon. $\boldsymbol{j}$, Uneven section thickness. $\boldsymbol{k}$, Enclosure of the ultramicrotome usually solves most issues that cause uneven section thickness.

segmentation are desired to speed the reconstruction process. As these and other approaches are optimized, the time required to analyze brain ultrastructure and connectivity should decrease markedly. sSTEM is unsurpassed for high resolution three- dimensional reconstruction in brain neuropil; hence, the effort to produce high-quality serial thin sections will be rewarded with beautiful images of brain ultrastructure that can be accurately quantified.

\section{References}

Bang BH, Bang FB (1957) Graphic reconstruction of the third dimension from serial electron microphotographs. J Ultrastruct Res 1:138-139.

Birch-Andersen A (1955) Reconstruction of the nuclear sites of Salmonella typhimurium from electron micrographs of serial sections. J Gen Microbiol 13:327-329.

Briggman KL, Denk W (2006) Towards neural circuit reconstruction with volume electron microscopy techniques. Curr Opin Neurobiol 16:562-570.

Gray EG (1959) Axo-somatic and axo-dendritic synapses of the cerebral cortex: an electron microscopic study. J Anat 93:420-433.

Jensen FE, Harris KM (1989) Preservation of neuronal ultrastructure in hippocampal slices using rapid microwave-enhanced fixation. J Neurosci Methods 29:217-230.

Knott GW, Holtmaat A, Wilbrecht L, Welker E, Svoboda K (2006) Spine growth precedes synapse formation in the adult neocortex in vivo. Nat Neurosci 9:1117-1124.

Ostroff LE, Fiala JC, Allwardt B, Harris KM (2002) Polyribosomes redistribute from dendritic shafts into spines with enlarged synapses during LTP in developing rat hippocampal slices. Neuron 35:535-545.

Palay SL, Palade GE (1955) The fine structure of neurons. J Biophys Biochem Cytol 1:69-88.

Spacek J, Lieberman AR (1974) Ultrastructure and three-dimensional organization of synaptic glomeruli in rat somatosensory thalamus. J Anat (Lond) 117:487-516.

Stevens JK, Davis TL, Friedman N, Sterling P (1980) A systematic approach to reconstructing microcircuitry by electron microscopy of serial sections. Brain Res Rev 2:265-293. 